\title{
Tangence
}

\section{Proposition pour une lecture de poésies francophones}

\section{Fernando Lambert}

Numéro 49, décembre 1995

Les littératures francophones de l'Afrique et des Antilles

URI : https://id.erudit.org/iderudit/025874ar

DOI : https://doi.org/10.7202/025874ar

Aller au sommaire du numéro

Éditeur(s)

Tangence

ISSN

0226-9554 (imprimé)

1710-0305 (numérique)

Découvrir la revue

Citer cet article

Lambert, F. (1995). Proposition pour une lecture de poésies francophones.

Tangence, (49), 9-22. https://doi.org/10.7202/025874ar d'utilisation que vous pouvez consulter en ligne.

https://apropos.erudit.org/fr/usagers/politique-dutilisation/ 


\section{Proposition pour une lecture de poésies francophones}

\section{Fernando Lambert}

Dans le monde de l'enseignement, aussi bien de la part des élèves ou des étudiants que de la part de trop de professeurs, la poésie est souvent l'objet d'un préjugé défavorâble. On semble considérer qu'elle est réservée au petit nombre, qu'elle n'est pas accessible à tout lecteur normal. Cette réserve s'accroît encore lorsqu'il s'agit de poésies francophones. Passe encore pour la poésie française et pour la poésie québécoise. Mais lorsque l'on parle de poésie africaine ou de poésie antillaise, la première réaction est de croire que cette production, fût-elle de langue française, ne peut nous rejoindre et nous parler au plus intime de notre être.

Vouloir présenter les poésies francophones à des étudiants d'une autre culture que celle du poète relève-t-il vraiment d'un défi? Une expérience souvent répétée auprès de jeunes lecteurs québécois montre au contraire que le défi n'est que de surface. Pour les poètes qui utilisent la langue française et qui n'appartiennent pas à notre culture, la relation à la poésie est la même que pour tout poète français ou québécois, elle est avant tout une relation au langage, à un langage spécifique qui tire parti de pouvoirs particuliers de la langue: rythme, sonorité ou musicalité, pouvoir d'évocation ou d'incantation, densité et même opacité. Comme dans toute poésie, peu importe la langue de création, ce langage recourt à une forme privilégiée, l'image poétique.

\section{Où se situe la différence?}

La différence entre les poésies francophones ne réside pas dans la nature même de la poésie. Elle ne se trouve pas non plus, du moins pas de façon essentielle, dans la relation à la langue française qui évidemment est différente selon que le français est langue maternelle ou langue officielle comme dans les pays qui ont été des colonies de la France. On sait en effet que les poètes africains ou antillais, comme tous les écrivains de ces mêmes pays, ont développé une maîtrise souvent raffinée de la langue 
française. Ce qui fait vraiment la différence, c'est la vision du monde, c'est le système de référence, c'est la culture propre de chaque poète francophone.

Chaque poésie francophone devient ainsi un lieu de découverte de l'être humain dans sa proximité et dans sa différence avec le lecteur, comme la langue française devient l'instrument de communication entre chaque poète et ses lecteurs, permettant d'établir des ponts entre divers univers culturels.

\section{Y a-t-il une méthodologie de la différence?}

L'intérêt pour les poésies francophones peut être facilement éveillé. Les voies pour le faire sont nombreuses, à commencer par la lecture à haute voix de quelques poèmes bien choisis. C'est là un appât utile. Il est possible et surtout nécessaire de pousser plus loin. Bien entendu, toute poésie étant langage, les diverses méthodologies qui permettent d'étudier le langage, peuvent être mises à contribution: sémantique, sémiologie, rhétorique, etc. Certaines cependant semblent plus appropriées, si l'objectif visé consiste à faire lire la spécificité de la poésie de chaque poète, la particularité de son langage et l'originalité de son univers. L'orientation ainsi dessinée prend en compte tout autant le contenu que la forme de cette poésie. Elle considère autant les structures internes au poème et au recueil que le système référentiel dont l'un et l'autre sont porteurs. Texte et contexte, c'est une excellente façon de faire entrer le lecteur dans cette poésie.

Les pistes de lecture ne manquent pas pour introduire à ces poésies selon la perspective proposée. Il y a une de ces pistes qui se révèle particulièrement pertinente à l'ensemble des poésies francophones: c'est la relation du poète francophone à l'histoire et sa manifestation à travers les poèmes. Elle convient en effet tout aussi bien pour la poésie québécoise, que pour la poésie maghrébine, pour la poésie africaine, pour la poésie antillaise et pour d'autres encore. En mettant en présence ces diverses poésies; il devient possible de développer une lecture comparée qui permette une expérience littéraire et humaine fort riche et qui ouvre sur des univers culturels nouveaux. 


\section{Histoire et poésie : aventure collective et aventure per- sonnelle}

Pourquoi privilégier l'histoire qui est un bien collectif alors que les nombreuses poésies francophones sont le fait de créations individuelles? En effet, chacun de ces poètes écrivant et publiant en français s'intègre à un système, celui de la lecture qui bien entendu peut être orale mais aussi personnelle et silencieuse. La poésie une fois publiée se donne à lire à tout lecteur. Comment la poésie permet-elle à un poète d'inscrire une aventure et une histoire collectives dans sa parole poétique qui est intimement liée à son aventure personnelle? Que nous permet de lire la relation du poète à l'histoire? Que nous apprend cette relation sur la culture de son auteur?

C'est la démonstration que nous voulons faire ici en parcourant quelques recueils de poésie. Le premier est du Martiniquais Édouard Glissant, Les Indes, publié en 1955 et réédité en $1965^{1}$. Les deux autres sont du poète sénégalais Léopold Sedar Senghor, Chants d'ombre publié d'abord en 1945 et Éthiopiques paru en $1956^{2}$. Ces poètes représentent deux espaces francophones, les Antilles et l'Afrique, reliés par l'histoire et pourtant très différents parce que l'histoire bifurque à un moment donné, à cause de la rencontre avec d'autres parcours.

\section{L'histoire et la poésie d'Édouard Glissant ou l'histoire comme métaphore}

Comment un Martiniquais peut-il voir l'histoire dans laquelle il a été placé par le hasard des événements? Il appartient à un peuple qui compte dans son passé un déracinement dramatique de sa terre maternelle, l'Afrique, et un réenracinement dans un autre espace, les Antilles. Pour compliquer les choses, il se retrouve dans un lieu où se sont croisés aussi bien biologiquement que culturellement des hommes d'origines diverses. Sa culture a ainsi été obligée d'intégrer dans une réalité nouvelle, l'antillanité puis la créolité, les apports culturels de plusieurs communautés humaines : caraïbéenne, européenne, africaine, asiatique.

1 Édouard Glissant, Poèmes, Un champ dîles, La terre inquiète, Les Indes, Paris, Seuil, 1965.

2 Léopold Sedar Senghor, Fuvre poétique, Paris, Seuil, coll. "Points ", 1990. 
12

Lorsque le poète Édouard Glissant veut dire son aventure et l'aventure de son peuple, il transforme l'histoire en une grande métaphore qui prend une dimension épique et en vient à couvrir toute expérience humaine. Dans l'histoire occidentale, la naissance des Antilles a comme origine le voyage de Christophe Colomb en Amérique, en 1492. Comme dans toute histoire coloniale, c'est habituellement le blanc qui amène l'autre à l'existence et à la civilisation. Mais on sait aussi que l'origine dont il est ici question est tout à fait mythique. Les Antilles et l'Amérique n'étaient pas des territoire vierges en 1492. Elles étaient propriétés de populations que l'on a nommées indiennes, les intégrant ainsi au grand rêve du blanc. Que l'on se souvienne du malaise suscité dans les Antilles et auprès des descendants des premiers habitants, par la célébration du cinquième centenaire de la découverte de l'Amérique, en 1992.

Le poète martiniquais construit son recueil, Les Indes, sur la quête d'une nouvelle route des Indes par les européens du $\mathrm{xv}^{\mathrm{e}}$ siècle. Christophe Colomb est chargé de trouver cette route plus courte de l'or, des épices et de la soie. C'est en fait une tout autre route qui va être ouverte, celle de la conquête de l'Amérique et en cours d'histoire, l'Afrique est entraînée au prix de plusieurs de ses fils et filles dans cette grande aventure.

Le recueil comprend six chants et chaque chant s'ouvre par une "péripétie" ou par l'événement marquant d'une phase de l'épopée de la quête des Indes. Dans le premier chant, c'est le départ de Gênes. Christophe Colomb incarne tous les grands découvreurs et aussi tous les hommes qui ont voulu vivre leur rêve. Nous glissons rapidement de l'histoire dans la métaphore: "L'imagination crée à l'homme des Indes toujours suscitees, que l'homme dispute au monde" ${ }^{3}$. Dans le port en fête, Colomb et ses hommes éprouvent tout de même la peur: "... le rêve s'épuise dans son projet. L'homme a peur de son désir, au moment de le satisfaire " ${ }^{4}$. Tout au long de son aventure, l'homme est toujours confronté à la dualité exprimée ici par le couple, y compris le couple de Vérone, Roméo et Juliette, et son projet d'un amour impossible. Le poète en vient à dire qu'il existe "deux Indes, deux levures d'or saignant" (p. 88). Il y a les Indes rêvées, il y a les Indes réalisées. Bien entendu, l'écart est

3 Édouard Glissant, op. cit., p. 77.

4 Ibid. 
grand entre le rêve et la réalité. Mais les Indes sont "levures", elles font lever le rêve, elles le gonflent jusqu'à sa maturité, jusqu'à ce qu'il devienne une force qui provoque le départ. Et il ajoute: "Mais les Indes sont vérité". On doit les écouter.

Il faut alors partir. Et c'est le voyage dont la fonction initiatique est explicite. En effet, la route, c'est la mer et la mer est dite savante. Il est même question des "Deux Livres d'azur saignant" 5 . Le livre est bien une métonymie du savoir, de la science. Ces deux livres se révèlent être la mer bleue et le ciel bleu. Le ciel apprend beaucoup de choses au marin, puisque c'est par l'observation des étoiles que ce dernier peut se guider. La mer aussi est une source de savoir: elle apprend la prudence, la patience, l'humilité, le dépouillement et beaucoup d'autres valeurs encore.

Le deuxième chant est consacré au voyage vers les Indes, vers le rêve. La longue et dure traversée de trois mois est marquée par la peur, car le voyage est l'"ascèse "nécessaire pour être digne d'un sable nouveau, d'une rive nouvelle. Il faut franchir la " ténébre entre la Demeure et la Connaissance: l'inépuisable Voyage " 6 . Plus prosaïquement, il faut quitter le confort du foyer, du connu et chausser les sandales ou prendre la mer pour aller quérir la connaissance. On doit accepter de laisser des choses derrière soi, en sacrifier d'autres en cours de route pour aller vers de nouvelles. Lorsqu'on nourrit et poursuit un rêve, peut-on faire autrement? "Mais qui peut, ô marins, se déprendre des Indes?" 7. Dans ce deuxième chant, tout au long de ce voyage, il est beaucoup question de temps: "Passe le temps, quand l'homme est mousse de l'attente..." ${ }^{8}$. Il y a aussi ceux qui meurent pendant la traversée, morts avec leur rêve entre deux rives, et que l'on confie à la mer, les faisant passer d'une solitude à une autre. Le voyage s'achève dès qu'on touche l'autre rive et que l'on débarque sur une plage que l'on dit "vierge" .

Après avoir rêvé les Indes, après l'attente de la traversée, la terrible réalité: la conquête du nouveau monde. Les principaux faits historiques de cette conquête sont repris dans ce troisième chant où le fusil va de paire avec la croix. On baptise et on tue,

$\begin{array}{ll}5 & \text { Ibid., p. } 96 . \\ 6 & \text { Ibid., p. } 97 . \\ 7 & \text { Ibid., p. } 101 . \\ 8 & \text { Ibid., p. } 106 . \\ 9 & \text { Ibid., p. } 112 .\end{array}$


14

en toute tranquillité de conscience puisqu'avant la mort, on a assuré le salut. Le capitaine à cheval, à la fois conquérant et prêtre, parcourt ce nouveau monde, cette nouvelle terre qu'il aime. Cette terre est femme. C'est donc le langage amoureux qu'il utilise pour la conquérir. Il veut être l'unique amant de cette terrefemme, belle, riche. Il élimine tous les autres amants pour être le seul à la posséder.

L'homme se prend alors pour un dieu: "L'homme est ce dieu, quand il tue l'homme ou l'humilie d'eau sainte..." ${ }^{10}$. Il peut alors tout se permettre. Il pille les mines d'or et de métaux précieux. Il saccage l'espace tant physique que spirituel. Il massacre les populations locales: les autochtones meurent dans le travail inhumain des mines. L'appétit des conquérants s'étend bien au-delà des îles, des Antilles, jusqu'à l'ensemble du continent américain. Le capitaine intègre dans la figure du conquérant, les Pizzaro, les Cortez. Même si ce nouveau monde n'est pas les Indes: "Inde je te dirai. Inde de l'Ouest: afin que je regagne mon rêve " ${ }^{11}$. Le mouvement lancé ne peut être arrêté.

Après avoir dépeuplé le nouveau monde, il faut donc le repeupler pour que le rêve continue. Avec le quatrième chant, les Indes de rêve se transforment en l' "Inde de souffrance " ${ }^{12}$. C'est la période de la traite des Noirs qui commence et elle a duré quelques siècles. "La réalité est fille de l'homme vraiment: née des contradictions qu'il a vécues et suscitées" 13 . Une nouvelle rive, l'Afrique, en face du nouveau monde, sur la route du soleil, d'est en ouest, par-delà l'océan. Pour quelles Indes les Africains qu'on déporte de l'Afrique partent-ils? Dans les cales des négriers sur la mer. La vie de l'esclave à bord, comme celle d'un bétail dont on ne veut pas abîmer la valeur. Le silence de la mer. "L'homme accomplit son océan; il râle la mer " ${ }^{14}$. Chaque esclave prisonnier a ses réactions. L'un échappe aux gardes chiourmes et se jette à la mer. L'autre pense aux siens, à sa femme qui est à bord et qu'il ne peut voir. Pour tous, "les Indes sont marché de mort " ${ }^{15}$. Tout au long de ce chant, ce trafic humain se fait sous la

10 Ibid., p. 131.
11 Ibid., p. 128.
12 Ibid., p. 135.
13 Ibid.
14 Ibid., p. 141.
15 Ibid., p. 143.


lumière du soleil, en plein jour, sur la route du soleil, du levant à son couchant. Et quelques siècles plus tard, maintenant ou le présent du poète, sur la rive du nouveau monde, "l'adolescent guetteur du futur" qui peut dire: "Nous sommes fils de ceux qui survécurent" " 16 .

Le cinquième chant nous parle précisément des "fils des survivants", de leurs souffrances, de leur histoire, de leur révolte. Cette phase est celle des "Indes déchirées"17. À leur tour, ces descendants découvrent la terre où on les a transplantés, où ils ont connu les souffrances de l'esclavage. Ils se mettent à aimer cette terre qui pour eux aussi devient femme, provoquant une relation amoureuse qui les conduit à la révolte contre le maître pour la possession de leur terre. Car l'Inde aussi est femme, terre du rêve. Ils s'abandonnent à leur tour à leur rêve: "L'Inde est imaginaire, mais sa révélation ne l'est pas..." ${ }^{18}$. Les figures des grands héros antillais et haïtiens sont alors convoquées: Delgrès qui a tenu la Guadeloupe pendant trois ans contre les Européens; Toussaint Louverture qui a pris les armes pour son peuple et qui, trahi, est obligé de retraverser l'océan pour aller mourir dans l'hiver, au fort de Joux; Dessalines, son lieutenant, qui n'a connu de toute sa vie que les guerres, destin qui l'a amené à semer la mort. Puis viennent ces autres transplantés, "les fils d'Orient qui font de la sagesse leur obole " ${ }^{19}$. Il s'agit de ceux que l'on continue à appeler les Coolies, ces Asiatiques amenés dans les îles au moment de l'abolition de l'esclavage et qui n'ont pu retourner dans leur pays. Les luttes de libération et tous les morts engendreront la Liberté. Les Antilles viennent à nouveau à l'existence: "Terre née d'elle-même, pluie des Indes assumées " 20.

Le poème, Les Indes, s'achève avec le retour sur la rive du départ, dans le sixième chant. C'est bien entendu du retour de Christophe Colomb à Gênes dont il est d'abord question. Mais celui-ci a eu des précurseurs: Marco Polo, Vasco de Gama, Magellan. Eux aussi ont poursuivi leur rêve sur les mers, vers des "Indes rêvées". Mais la grande question est de savoir qui revient, ce qu'il a appris, ce qui a grandi en lui. Que rapporte-t-on de 
16

cette quête des Indes? Et après eux, d'autres sont partis et d'autres partiront. Pourquoi?

Mais peut-être enfin l'homme n'a-t-il que même désir et même ardeur, n'importe soit-il? Et d'où qu'il vienne, même souffrance connaissable? Quelles Indes l'appellent? Ou, si son rêve n'est déjà qu'une passionnée raison, quel océan pourtant s'impose entre elle et lui? - Nul ne peut dire en certitude; mais chacun tente la nouvelle traversée! La mer est éternelle. ${ }^{21}$

La lecture proposée ici de ce long poème en six chants que constitue le remarquable recueil d'Édouard Glissant, Les Indes, n'épuise pas toute la richesse de cette poésie. La métaphore que le poète tire du voyage et du projet de Colomb, est l'occasion de faire revivre en quelques touches essentielles l'histoire de la Martinique et des Antilles. Mais l'aventure des Martiniquais s'inscrit dans quelque chose de plus large, dans l'histoire de l'Europe et dans l'histoire de l'Amérique. Le poète et son peuple partagent le sort de tous les opprimés: Africains, Caraibes, "Coolies" ou Indiens, Amérindiens, etc. Le Martiniquais comme les Antillais, rassemble en lui-même l'héritage biologique, humain, culturel et religieux de tous les peuples qui se sont croisés dans les îles. Ses luttes pour la liberté lui ont permis de s'inscrire dans l'histoire et d'apporter sa part à l'homme. C'est ce passé et cette réalité qui le constituent et qu'il doit assumer dans leur complexité. C'est alors qu'il trouve sa véritable place et dans l'histoire, et parmi les autres hommes. Pour lui, insulaire, davantage que pour les autres, "la mer est éternelle"22. Elle est constamment présente, nourrissant l'imagination, invitant au départ, à la poursuite de quelque rêve, à la recherche de nouvelles Indes.

La poésie est vérité profonde. Elle est aussi métamorphose par la vision et par le langage. Elle permet de voir et de dire les choses essentielles. Édouard Glissant, dans ce recueil, réussit merveilleusement à nous en convaincre. Jean Paris a dit de ce poème: "Avec Les Indes, soudain, le poète élève la voix la plus puissante qu'on ait entendue depuis Saint-John Perse". 


\section{L'histoire et la poésie de L. S. Senghor ou l'histoire comme lieu de réintégration et de réappropriation}

Le poète sénégalais Léopold Sedar Senghor entretient avec l'histoire une relation bien différente de celle du poète martiniquais Édouard Glissant. Rappelons ce qu'Aimé Césaire aime rapporter de ses premières rencontres avec le jeune Senghor. Ils se sont connus à Paris, en 1929, au Lycée Louis le Grand. Césaire dit qu'il était étonné presqu'indisposé d'entendre ce "petit sérère" l'expression est de Césaire - parler avec autant d'assurance et d'insistance de son pays, de sa culture, du passé de son peuple. Césaire avait quitté son île natale, la Martinique, avec l'espoir de n'y jamais revenir, laissant apparemment toute la place à une assimilation la plus large possible. Heureusement le Cabier d'un retour au pays natal (1939) vient prouver le contraire. Le jeune Senghor, de son côté, affirme dès le début ses références historiques, culturelles et africaines. Cela ne l'empêche pas de se laisser séduire pendant un bon moment par l'assimilation au monde français et au modèle occidental. Mais le moment venu, il peut rebondir et revenir à ses sources africaines.

Dans les faits toutefois, Senghor s'est vite rendu compte que ses connaissances sur l'Afrique étaient très élémentaires. Ce que son enfance lui avait appris du passé du peuple sérère se réduisait à quelques noms et à quelques événements dont plusieurs appartenaient plus au mythe qu'à la réalité historique. Par ailleurs, l'histoire de l'Afrique n'avait pas encore été écrite, du moins pas par les Africains eux-mêmes. Senghor lit cependant tous les documents disponibles, même si ceux-ci ont été écrits par des administrateurs coloniaux, d'abord sur le pays sérère, son pays, et sur le passé de l'Afrique. On peut ainsi constater la différence essentielle entre ses premières références à l'histoire africaine dans les premiers poèmes qu'il a écrits et que nous retrouvons sous le titre Poèmes perdus, dans la dernière réédition de ses poèmes (1990) où on trouve de timides références aux amazones du Dahomey, aux Guelwars mandingues, à Tombouctou, à la déesse tunisienne Tanit, et celles qui nourrissent les poèmes de Chant d'ombre (1945) où sur les voies de la Négritude, se déploient les grandes images senghoriennes, dans toute leur force et leur éclat.

Chants d'ombre se présente comme un recueil essentiel à la compréhension de la poésie de Senghor, car il construit en tout premier lieu la géographie et l'histoire du poète. C'est d'abord les 
18

lieux et le temps de l'enfance du poète qui fondent l'univers poétique senghorien. La première figure qu'il retient de l'histoire sérère est une figure qui se situe dans le temps personnel du poète, puisqu'il s'agit de la grande figure royale de Koumba N'Dofène Diouf ${ }^{23}$, dernier roi légitime du royaume du Sine, ami de son propre père et dont Senghor enfant a vu les fastes et les attributs royaux: les chevaux, le manteau de pourpre, les dyoundyouns ou tambours royaux, les échanges de cadeaux et d'énigmes, etc.

De l'histoire immédiate du dernier roi légitime sérère, le poète convoque l'histoire des royaumes sérères, le royaume du Sine et le royaume du Saloum, dont les sources historiques, au moment de l'écriture des poèmes de Chants d'ombre, relèvent encore pour une très large part de récits mythiques. Mais le poète ne distingue pas histoire orale et histoire à l'occidentale, cette dernière ne considérant que les documents écrits. Ce sont les figures des fondateurs de ces royaumes qui lui importent: "l'Éléphant de Mbissel " ${ }^{24}$ ou Maïsa Wali Dione, roi mandingue venu d'Élissa du Gâbou et ayant établi la première capitale du royaume du Sine à Mbissel et sa sœur Sîra-Badral, fondatrice du royaume voisin du Saloum et figure de la mère du peuple sérère, identifiée rapidement à la figure de la terre-mère ${ }^{25}$. C'est l'origine de la dynastie des Guelwars qui a régné sur le pays sérère depuis le XIV $^{\mathrm{e}}$ siècle, que le poète reprend et, dans un glissement très senghorien, il en fait l'origine même de son peuple. Il fait sienne leur chevauchée épique depuis Élissa du Gâbou jusqu'au pays sérère et la résistance grâce à ses rois, du peuple sérère devant les tentatives d'invasion de la part des Almamy ou chefs musulmans des régions limitrophes.

Ce qui est éclairant, c'est la façon dont le poète se rattache dans un premier temps à cette histoire du pays sérère, puis ensuite à l'histoire de l'Afrique. Pour le poète, comme pour tout Africain, ce qui est essentiel, c'est de pouvoir se relier à une généalogie. Lui, le fils du commerçant Basile Diogoye Senghor, se rattache à l'histoire des rois de son pays, à l'Afrique aristocratique donc. Son intégration se fait par le procédé de l'identification: "J'étais moi-même le grand-père de mon grand-père / J'étais son

23 Léopold Sedar Senghor, op. cit., p. 31.

24 Ibid., p. 49.

25 Ibid., p. 34. 
âme et son ascendance, le chef de la maison d'élissa du Gâbou..." ${ }^{26}$; "Kaya-Magan je suis! la personne première...."27. Descendant donc du fondateur du royaume du Sine et aussi fils de Kaya-Magan, le Roi de l'or, empereur du Ghana, région qui a été pendant longtemps connue par la suite comme la Côte d'Or.

Le mouvement de rattachement du poète à l'Afrique s'élargit donc du pays sérère à l'histoire du continent. C'est d'abord Gongo-Moussa ${ }^{28}$ ou Kankan-Moussa, l'un des successeurs de Soundjata ( $\mathrm{XrV}^{\mathrm{e}}$ siècle) à la tête de l'empire du Mali et qui a porté l'empire à son apogée au $\mathrm{XVI}^{\mathrm{e}}$ siècle. L'histoire a conservé de cet empereur le pèlerinage digne de sa grandeur et de sa magnificence qu'il fit à La Mecque. Mais le poète ne se rattache pas qu'à l'Afrique des richesses et aux grandes figures, il se reconnaît aussi dans l'Afrique des souffrances, d'une part, dans la Traite des noirs qui a marqué des siècles sombres de l'histoire africaine, et d'autre part, au-delà de l'Afrique, dans le sort fait à ses frères de la diaspora, ses frères noirs américains, à qui il a dédié son magnifique poème, "À New York" ${ }^{29}$. Il réclame encore pour lui les docteurs de Tombouctou, la sagesse des Keïta ${ }^{30}$, héritiers de Soundjata Keita, le fondateur de l'empire du Mali. Le poète, fils authentique de l'Afrique, peut se faire alors le légitime porte-parole de cette Afrique, son "dyali ", son griot, son chantre.

Autre trait significatif du rattachement du poète à l'Afrique: dès son troisième recueil, Éthiopiques, les grandes figures de l'histoire africaine se transforment en symboles du poète lui-même ou de son univers poétique. Il ne se contente plus alors de réclamer ces figures comme siennes, il se les approprie comme images poétiques. Le Chaka historique, chef de l'empire zulu, devient en fait le double de Senghor, président-poète. La problématique du second, qui en fait n'a jamais choisi entre ses responsabilités politiques et sa poésie, devient celle du premier qui, lui, aurait sacrifié la poésie au pouvoir, sans en retirer les résultats escomptés puisqu'il meurt de la main de ses demi-frères et rivaux. De même, la Reine de Saba qui dans l'univers poétique de Senghor prend rang parmi les figures nobles et les figures d'autorité, 
20

devient de plus l'une des grandes figures de la négritude dans ses échanges avec une autre culture, la culture sémite, amorçant le mouvement d'échanges avec l'Europe puis avec le monde.

Cette lecture cursive de deux des trois premiers recueils de Senghor met très clairement en évidence que le poète donne une place prépondérante à l'histoire de l'Afrique et qu'il privilégie cette relation parce qu'elle le réintègre symboliquement à cette Afrique. Mais le parcours poétique de Senghor s'inscrit aussi dans l'histoire personnelle du poète qui a poursuivi ses études dans la métropole, a enseigné à Tours et à Paris, a participé à la Guerre 1939-1945, a été député africain à l'Assemblée nationale française, puis membre du cabinet d'Edgar Faure, devenant enfin président du Sénégal en 1960. Ses premiers recueils de poèmes ont été écrits en France et l'histoire européenne, particulièrement française y a une place, mais à un rang secondaire, sauf dans le recueil Hosties noires (1948), consacré aux combattants noirs de la deuxième Grande Guerre. Il est donc normal que sa relation à l'histoire soit beaucoup plus complexe si l'on considère l'ensemble de son œuvre poétique qui s'étend des années trente aux années quatre-vingt.

\section{Édouard Glissant et Senghor, les Antilles et l'Afrique en regard de l'histoire}

Dans une démarche qui se voulait au départ pédagogique, trois recueils, l'un d'Édouard Glissant, les deux autres de Senghor, recueils publiés aux alentours des années cinquante, ont été mis en présence de façon ambitieuse sans doute, pour tracer des pistes de lecture à l'intention de lecteurs francophones mais de culture différente de la culture martiniquaise et de la culture africaine. C'est la relation à l'histoire de chacun de ces poètes qui a guidé la lecture. Autre critère du choix des poètes mis en regard l'un de l'autre: Senghor est né en 1906, Glissant, en 1928. Cette lecture en parallèle conduit à certaines observations qui tiendront lieu de conclusion.

Ces recueils montrent jusqu'à quel point les positions face à l'histoire peuvent être différentes chez des poètes que l'on aurait pu considérer comme ayant tout normalement beaucoup en commun. Cela établit de façon claire l'importance de l'histoire pour un peuple et pour chacun de ses membres. C'est en regard de l'histoire, on le sait, que se définissent principalement l'identité 
collective et l'identité individuelle. Dans le cas de Glissant et de Senghor, ce n'est pas une question de génération qui fait la différence, mais le fait que les Antilles ont connu une histoire beaucoup plus traumatisante que celle qu'a connue l'Afrique, les deux partageant pourtant l'expérience coloniale. Visiblement, l'Afrique offre à Senghor des points de repère auxquels il lui est facile de s'identifier, alors que les Antilles, issues d'un brassage beaucoup plus complexe, présentent à Glissant une réalité plurielle qu'il est énormément plus difficile d'assumer.

Les références à l'histoire permettent de prendre conscience de la différence très grande entre l'univers poétique du poète sénégalais et celui du poète martiniquais. Non seulement chacun se réfère à des événements et à des héros différents, mais ces derniers s'inscrivent dans des lieux et un temps qui créent une distance signifiante et génératrice de deux mondes. Nous avions été habitués de voir pendant longtemps la part africaine des Antillais. Césaire, l'un des pères de la Négritude avec Senghor et le Guyanais Léon Gontran Damas, ont redonné la place qui lui revient, à cette composante de l'être antillais, part d'autant plus difficile à assumer qu'elle oblige à intégrer les événements tragiques de la traite et de l'esclavage. Glissant ne rejette pas cette part africaine. Il réclame en fait la totalité de son être, son antillanité qui fait de lui un métis dans toute la richesse de cet être nouveau. L'Antillais est la somme des valeurs humaines et culturelles qui le font différent, tout en le situant dans l'histoire du nouveau monde.

Senghor, de son côté possède des bases ontologiques, culturelles et historiques qui lui permettent d'établir une dialectique fort différente entre l'histoire de l'Afrique et l'histoire de l'Occident. Pour le poète sénégalais, c'est l'Afrique qui est posée comme point de départ, comme référence constante et c'est à l'Afrique qu'il revient toujours, dans cet échange très senghorien, au fond très humain, et qui n'existe que dans la mesure où cet échange est le "rendez-vous du donner et du recevoir", comme le redit le poète tout au long de son ourvre.

Toutefois, Senghor et Glissant se rejoignent pleinement dans le mode de relation et d'expression qu'est la poésie. Nous nous retrouvons en présence de deux grands poètes. La lecture de leurs poèmes nous en convainc. Tous les deux possèdent à un haut degré le sens et la force de l'image poétique. Ils maîtrisent 
22

magnifiquement le langage poétique dont la cohérence et la puissance d'évocation créent un univers qui engage en fait tout l'homme. L'un et l'autre nous font partager profondément leur vision et leur monde.

La piste de lecture que constitue la relation d'un poète à l'histoire se révèle donc très féconde. On pourrait la pratiquer avec autant de succès dans l'œuvre poétique de poètes québécois, par exemple dans l'Ode au Saint-Laurent du regretté Gatien Lapointe. Cette piste n'est en fait qu'une porte d'entrée dans les poésies francophones, car elle ouvre en fin de compte sur ce qui constitue chacune de ces poésies. 